\title{
Variabilidade espacial de atributos químicos de um Latossolo Vermelho-Amarelo cultivado em plantio direto ${ }^{1}$
}

\author{
Spatial variability of chemical attributes of a Red-Yellow Latosol under no-tillage \\ cultivation
}

\author{
Julião Soares de Souza Lima ${ }^{2 *}$, Samuel de Assis Silva ${ }^{3}$ e José Marcilio da Silva ${ }^{4}$
}

\begin{abstract}
RESUMO - Objetivou-se avaliar a extensão e a intensidade da dependência espacial da fertilidade de um Latossolo Vermelho-Amarelo distrófico, cultivado sob plantio direto, utilizando, de forma conjunta, técnicas de geoestatística e estatística multivariada. $\mathrm{O}$ experimento foi conduzido em uma área cultivada com soja em plantio direto no município de Rio Verde - GO. O solo foi amostrado nas profundidades de 0-0,05 m e 0,05-0,15 m, em uma malha amostral com 44 pontos. Os atributos avaliados foram $\mathrm{pH}$ em $\mathrm{CaCl}_{2}, \mathrm{Ca}, \mathrm{Mg}, \mathrm{Al}, \mathrm{P}, \mathrm{K}, \mathrm{H}+\mathrm{Al}, \mathrm{CTC}, \mathrm{SB}, \mathrm{V}$ e MO. Os dados foram analisados por meio da análise da estatística descritiva seguida de uma análise de componentes principais de forma a gerar componentes que expliquem a maior parte da variação dos atributos. A geoestatística foi utilizada para verificar a existência e quantificar o grau de dependência espacial utilizando os componentes principais. A matéria orgânica apresentou maior continuidade espacial devido às características do sistema de plantio direto. De maneira geral, a área apresentou baixa fertilidade para o cultivo da soja, como diferente padrão de distribuição espacial entre camadas.
\end{abstract}

Palavras-chave: Geoestatística. Solo-manejo. Fertilidade do solo.

\begin{abstract}
The objective was to assess the extent and intensity of the spatial dependence of the fertility of a Red-Yellow Latosol, cultivated under a non-tillage system, by using both geo-statistical techniques and multivariate statistics. The experiment was conducted in an area planted with soybeans under a non-tillage system, in the city of Rio Verde, Goiás, Brazil. The soil was sampled at depths of $0-0.05 \mathrm{~m}$ and $0.05-0.15 \mathrm{~m}$, in a sampling grid with 44 points. The attributes evaluated were the $\mathrm{pH}$ of $\mathrm{CaCl} 2, \mathrm{Ca}, \mathrm{Mg}, \mathrm{Al}, \mathrm{P}, \mathrm{K}, \mathrm{H}+\mathrm{Al}, \mathrm{CEC}, \mathrm{BS}, \mathrm{V}$ and $\mathrm{MO}$. The data were analyzed using descriptive statistical analysis, followed by principal component analysis, in order to generate components which would explain most of the variations in the attributes. Geo-statistics was used to check and quantify the degree of spatial dependence, using the principal components. Multivariate analysis, associated with the geo-statistical analysis, showed satisfactory results for the spatial distribution of fertility. Organic matter showed greater spatial continuity due to the characteristics of the no-tillage system. In general, the area presented low fertility for the cultivation of soybeans, as well as different patterns of spatial distribution between layers.
\end{abstract}

Key words: Geostatistics. Soil management. Soil fertility.

\footnotetext{
*Autor para correspondência

${ }^{1}$ Recebido para publicação em 08/08/2011; aprovado em 10/06/2012

Parte da Tese de Mestrado do terceiro autor apresentada ao Programa de Pós-Graduação em Produção Vegetal/CCA/UFES

${ }^{2}$ Departamento de Engenharia Rural/CCA, Universidade Federal do Espírito Santo, Alto Universitário, Caixa Postal 16, Alegre-ES, Brasil, 29.500-000, juliao.lima@ufes.br

${ }^{3}$ Departamento de Ciências Agrárias e Ambientais, Universidade Estadual de Santa Cruz, Ilhéus-BA, Brasil, 45.662-900, samuel-assis@ hotmail.com

${ }^{4}$ Instituto Federal de Educação, Ciência e Tecnologia de Pernambuco, Campus de Barreiros, Barreiros-PE, Brasil, 55.560-000, marciliocilo@yahoo.com.br
} 


\section{INTRODUÇÃO}

A utilização de sistemas conservacionistas para a produção agrícola tem sido uma constante nos últimos anos. Tal constância está alicerçada, principalmente, na necessidade de sustentabilidade, preservação ambiental, melhoria da qualidade de solos agrícolas e redução dos custos de produção. O sistema de plantio direto (SPD), por exemplo, é eficiente para o atendimento de tais exigências, proporcionando aumento de rendimento dos sistemas produtivos, quando comparado aos sistemas de preparo convencional (TOPAKCI et al., 2011).

Devido à ausência de necessidade de operações de preparo periódico do solo, o SPD reduz os custos dos cultivos, em função da redução do tempo de trabalho e do consumo de combustível associado às etapas de plantio e semeadura (WANG; CHEN; RAHMAN, 2006). Além disso, a manutenção de palha sobre o solo favorece o controle da erosão, conservação da umidade, aumento da matéria orgânica (MO) e da atividade biológica, melhorando consequentemente a estabilidade dos agregados (AMADO et al., 2007; SILVA; REICHERT; REINERT, 2004).

Apesar das inúmeras vantagens que o sistema apresenta, tem-se observado problemas significativos de compactação do solo em áreas cultivadas sob plantio direto (AZEVEDO et al., 2007; SECCO et al., 2004; STRECK et al., 2004). Celik, Boydas e Altikat (2011) afirmaram que a ocorrência e a intensidade da compactação dependem da sequência de culturas, do sistema de manejo e da umidade do solo por ocasião do tráfego, sendo essa, de acordo com Wang, Chen e Altikat (2006), maior em solos argilosos.

Grego et al. (2006) caracterizaram o plantio direto como um sistema de elevada complexidade e que carrega igualmente, significativa variabilidade espacial, tanto para os atributos físicos quanto para os químicos do solo. Assim, como nos demais sistemas de cultivo, o conhecimento dessa variação é importante para o levantamento, manejo do solo e gerenciamento de práticas agrícolas (SILVA et al., 2010b).

Para analisar a estrutura da dependência espacial da fertilidade dos solos, muitos nutrientes devem ser analisados conjuntamente, o que, ao ser realizado por meio de métodos estatísticos univariados, eleva a dimensão do problema, além de onerar o processo (SILVA et al., 2010b). Uma opção para tais tipos de dados é o uso de análise multivariada, mais especificamente a análise de componentes principais (ACP), a fim de explicar a estrutura da variância e da covariância de um conjunto de variáveis, por meio da construção de combinações lineares dessas e da redução dimensional do fenômeno em estudo (JOHNSON; WICHNER, 2002).

Diversostrabalhostêmmostradoaspotencialidades do uso dessa técnica para o estudo de variáveis de solo
(GREGO; VIEIRA, 2005; LEÃO et al., 2007; MOTTA et al., 2002; SILVA et al., 2010b).

A fertilidade do solo é um fator decisivo para o rendimento satisfatório das culturas agrícolas e a sua distribuição espacial, horizontal e vertical, pode alterar significativamente a produtividade média em uma área cultivada. Nesse contexto, este trabalho teve por objetivo avaliar a extensão e a intensidade da dependência espacial da fertilidade de um Latossolo Vermelho-Amarelo distrófico, cultivado em sistema plantio direto, utilizando de forma conjunta técnicas de geoestatística e estatística multivariada.

\section{MATERIAL E MÉTODOS}

$\mathrm{O}$ experimento foi conduzido em uma área cultivada com soja em SPD, localizada no município de Rio Verde, Goiás, com coordenadas geográficas médias de $17^{\circ} 29^{\prime} 24^{\prime \prime} \mathrm{S}$ e $51^{\circ} 23^{\prime} 24^{\prime \prime}$ O. A região apresenta topografia plana, sendo a altitude média da área experimental de $784 \mathrm{~m}$. O clima é definido como tropical sazonal com temperatura média anual de $24{ }^{\circ} \mathrm{C}$.

$\mathrm{O}$ solo na área foi classificado como Latossolo Vermelho-Amarelo distrófico típico, textura argilosa (EMPRESA BRASILEIRA DE PESQUISA AGROPECUÁRIA, 2006), com valores médios das frações granulométricas de areia $=140 \mathrm{~g} \mathrm{~kg}^{-1}$, silte $=160 \mathrm{~g} \mathrm{~kg}^{-1}$ e argila $=700 \mathrm{~g} \mathrm{~kg}^{-1}$.

Construiu-se uma malha amostral com $2.200 \mathrm{~m}^{2}$, contendo 44 pontos amostrais. O espaçamento entre pontos foi variável, sendo de $5,0 \mathrm{~m}$ entre os 40 pontos centrais e 10,0 m entre os quatro extremos.

O SPD utilizado contemplou, nos últimos cinco anos, a sucessão da soja cultivada no verão, com o milheto cultivado na safrinha. $\mathrm{O}$ manejo de adubação da área foi baseado nos resultados da análise química do solo, sendo que um mês antes da semeadura da soja foi realizada uma adubação com cloreto de potássio $(\mathrm{KCl})$ na quantidade de $100 \mathrm{~kg} \mathrm{ha}^{-1}$, correspondente a $60,0 \mathrm{~kg} \mathrm{ha}^{-1} \mathrm{de}_{2} \mathrm{O}$, distribuídos a lanço. A adubação de semeadura foi realizada com o adubo MAP, compondo a fórmula 11-52-00 que foi aplicada na quantidade de $150 \mathrm{~kg} \mathrm{ha}^{-1}$, correspondente a 16,5 kg ha- ${ }^{-1}$ de nitrogênio (N) e 78,0 $\mathrm{kg} \mathrm{ha}^{-1} \mathrm{de}_{2} \mathrm{O}_{5}$, que foram distribuídos na linha de semeadura. A semeadura foi realizada no espaçamento de $0,50 \mathrm{~m}$ entre linhas, com estande de 240.000 plantas por hectare.

Após a colheita da soja, as amostras de solo foram coletadas nos pontos amostrais utilizando uma pá de corte reta, com a abertura de uma trincheira abrangendo a linha de plantio mais a metade do espaçamento das entrelinhas da cultura $(0,25 \mathrm{~m}$ de cada lado), totalizando $0,50 \mathrm{~m}$ de 
comprimento, $0,10 \mathrm{~m}$ de largura e $0,05 \mathrm{~m}$ de espessura, nas camadas de 0-0,05 m e 0,05-0,15 m.

As amostras de solo foram acondicionadas em sacos plásticos, identificadas e enviadas ao laboratório, onde foram submetidas às análises químicas de rotina de acordo com os procedimentos descritos pela Empresa Brasileira de Pesquisa Agropecuária (1997) para determinação dos seguintes atributos químicos: $\mathrm{pH}$ em $\mathrm{CaCl}_{2}$; $\mathrm{Ca}, \mathrm{Mg}, \mathrm{Al}, \mathrm{H}+\mathrm{Al}$, CTC e SB $\left(\mathrm{cmol}_{\mathrm{c}} \mathrm{dm}^{-3}\right)$; P e K $\left(\mathrm{mg} \mathrm{dm}^{-3}\right)$ e V $(\%)$. A MO $\left(\mathrm{g} \mathrm{kg}^{-1}\right)$ do solo foi determinada pelo método colorimétrico, conforme metodologia proposta por Raij et al. (2001).

A ACP foi realizada com base na matriz de correlação dos mesmos, de forma a identificar novas variáveis que explicam a maior parte da variação contida nos dados de origem. Em uma matriz original de dados, cada sítio tem um valor para cada atributo. Na ACP, cada sítio tem um valor para cada componente. Essas componentes podem ser vistas como "supervariáveis", construídas pela combinação da correlação entre as variáveis e são extraídas em ordem decrescente de importância em termos de sua contribuição para a variação total dos dados (KENT; COKER, 1992).

A seleção do número de componentes principais foi baseada no critério de análise da qualidade de aproximação da matriz de correlações, utilizando-se os componentes associados a autovalores superiores a 1 (JOHNSON; WICHERN, 2002). No caso da correlação dos componentes com os atributos químicos do solo, consideraram-se significativos os valores superiores a 0,7 , conforme Zwick e Velicer (1986).

A geoestatística foi utilizada para verificar a existência e, neste caso, quantificar o grau de dependência espacial utilizando os dados gerados por meio da aplicação da ACP. A análise foi conduzida a partir do ajuste de funções teóricas aos modelos de variogramas experimentais, com base na pressuposição de estacionaridade da hipótese intrínseca e conforme equação 1:

$$
\gamma^{*}(h)=\frac{1}{2 N(h)} \sum_{i=1}^{N(h)}\left[z\left(x_{i}\right)-z\left(x_{i}+h\right)\right]^{2}
$$

em que: $N(h)$ é o número de pares experimentais de observações $Z\left(x_{i}\right), Z\left(x_{i}+h\right)$, separados por um vetor $h$. No ajuste dos modelos teóricos aos variogramas experimentais determinaram-se os coeficientes efeito pepita $\left(\mathrm{C}_{0}\right)$, patamar $\left(\mathrm{C}_{0}+\mathrm{C}_{1}\right)$, variância estrutural $\left(\mathrm{C}_{1}\right)$ e alcance $(\mathrm{a})$ pelo software $\mathrm{GS}^{+}$, que utiliza o método dos mínimos quadrados e adota como critérios para a seleção o maior valor de $\mathrm{R}^{2}$ (coeficiente de determinação), a menor SQR (soma de quadrados dos resíduos) e o maior valor do coeficiente de correlação obtido pelo método de validação cruzada. Para análise do índice de dependência espacial
(IDE), foi utilizada a relação $\mathrm{C}_{0} /\left(\mathrm{C}_{0}+\mathrm{C}_{1}\right)$ e os intervalos propostos por Cambardella et al. (1994) que consideram a dependência espacial forte (IDE < 25\%); moderada $(25 \% \leq \mathrm{IDE}<75 \%)$ e fraca $(\mathrm{IDE} \geq 75 \%)$.

Comprovada a dependência espacial dos componentes principais utilizou-se o método de interpolação da krigagem ordinária para estimar valores em locais não medidos e construir os mapas temáticos.

\section{RESULTADOS E DISCUSSÃO}

Em alguns atributos observou-se a presença de pontos discrepantes (outliers). Esses valores discrepantes, na maioria dos casos, alteram os valores de medidas sensíveis a estas observações, como a média, amplitude, o desvio-padrão e a assimetria da distribuição dos dados. Quando não se observa influência desses valores, as análises subsequentes podem ser realizadas com a presença dos mesmos (LIBARDI et al., 1996). No trabalho em questão, a presença de tais valores não influenciou as medidas de posição, dispersão e a distribuição normal dos dados, optando-se pela sua manutenção nas séries de dados.

Considerando as duas profundidades, $81,8 \%$ dos atributos não apresentaram distribuição normal apesar da proximidade entre a média e a mediana (Tabela 1), bem como Cs e Ck próximos de zero. Entretanto, como afirmaram Isaaks e Srivastava (1989), a normalidade dos dados não é fundamental para aplicação da geoestatística, o que importa é que a distribuição dos dados não apresente caudas muito alongadas, o que foi observado nesses casos.

Segundo a classificação proposta por Warrick e Nielsen (1980), os coeficientes de variação apresentaram-se baixos (<12\%) para pH, MO e CTC e médios (CV entre 12 a $60 \%$ ) para os demais atributos nas duas profundidades. Em geral, os intervalos de CVs para os atributos estão em conformidade com os resultados encontrados por Amado et al. (2007) e, principalmente, por Carvalho, Silveira e Vieira (2002) em plantio direto nas camadas de 0-0,05 m e 0,05-0,20 m, em um Latossolo VermelhoEscuro distrófico, cultivando milho no verão e feijão no inverno, por um período de cinco anos.

Entre camadas, todos os atributos apresentaram diferença significativa pelo teste $t$, com exceção para o $\mathrm{Ca}$, o que pode estar relacionado com a não realização da calagem na área. A adubação de pré-semeadura realizada com $\mathrm{KCl}$ distribuído a lanço um mês antes da semeadura, contribuiu para maior concentração de K na superfície (0-0,05 m), com 1,7 vezes mais $\mathrm{K}$ em relação à camada de 0,05-0,15 m. O inverso ocorreu com o P, maior na camada mais profunda $(0,05-0,15 \mathrm{~m})$, na mesma proporção que o K. Isto é explicado pela distribuição 
Tabela 1 - Análise descritiva dos atributos químicos do solo nas camadas de 0-0,05 m e 0,05-0,15 m

\begin{tabular}{|c|c|c|c|c|c|c|c|c|c|}
\hline Atributo & Média & Mediana & Min, & Máx, & $S$ & $\mathrm{CV}$ & $\mathrm{Cs}$ & $\mathrm{Ck}$ & $\mathrm{DN}$ \\
\hline $\mathrm{pH}^{1}$ & $4,8 \mathrm{a}$ & 4,7 & 4,5 & 5,1 & 0,1 & 3,1 & 0,6 & $-0,1$ & $\mathrm{~ns}$ \\
\hline $\mathrm{pH}^{2}$ & $4,4 b$ & 4,4 & 4,1 & 5,0 & 0,2 & 4,5 & 0,6 & 0,3 & $\mathrm{~ns}$ \\
\hline $\mathrm{MO}^{1}$ & $39,8 \mathrm{a}$ & 39,8 & 32,7 & 45,9 & 3,3 & 8,3 & $-0,4$ & $-0,4$ & $*$ \\
\hline $\mathrm{MO}^{2}$ & $34,5 \mathrm{~b}$ & 34,0 & 28,8 & 44,4 & 3,4 & 8,3 & 0,8 & 1,1 & $*$ \\
\hline $\mathrm{P}^{1}$ & $3,9 b$ & 3,6 & 2,3 & 7,6 & 1,3 & 32,1 & 1,3 & 1,6 & Ns \\
\hline $\mathrm{P}^{2}$ & $6,6 a$ & 6,4 & 2,2 & 14,0 & 2,5 & 48,0 & 0,6 & 0,6 & $*$ \\
\hline $\mathrm{K}_{1}$ & $82,0 \mathrm{a}$ & 76,3 & 35,2 & 199,4 & 28,2 & 34,4 & 1,7 & 5,9 & $*$ \\
\hline $\mathrm{K}^{2}$ & $48,3 b$ & 43,0 & 19,6 & 105,6 & 18,4 & 38,0 & 0,9 & 1,2 & $*$ \\
\hline $\mathrm{Ca}^{1}$ & $1,5 \mathrm{a}$ & 1,5 & 1,1 & 2,7 & 0,3 & 21,1 & 1,3 & 3,0 & $*$ \\
\hline $\mathrm{Ca}^{2}$ & $1,4 \mathrm{a}$ & 1,4 & 0,6 & 2,4 & 0,5 & 32,9 & 0,4 & $-0,3$ & $*$ \\
\hline $\mathrm{Mg}^{1}$ & $0,9 a$ & 0,9 & 0,4 & 1,4 & 0,2 & 24,3 & 0,0 & 0,0 & $*$ \\
\hline $\mathrm{Mg}^{2}$ & $0,7 b$ & 0,6 & 0,2 & 1,4 & 0,3 & 39,5 & 0,8 & 0,8 & $*$ \\
\hline $\mathrm{Al}^{1}$ & $0,1 b$ & 0,1 & 0,0 & 0,2 & 0,0 & 55,7 & 1,1 & 2,35 & $*$ \\
\hline $\mathrm{Al}^{2}$ & $0,2 \mathrm{a}$ & 0,2 & 0,0 & 0,7 & 0,2 & 54,3 & 1,2 & 0,8 & Ns \\
\hline $\mathrm{H}+\mathrm{Al}^{1}$ & $5,3 b$ & 5,5 & 3,3 & 6,6 & 0,7 & 12,9 & $-0,7$ & 0,6 & $*$ \\
\hline $\mathrm{H}+\mathrm{Al}^{2}$ & $6,8 \mathrm{a}$ & 6,9 & 4,4 & 9,3 & 1,1 & 16,2 & 0,1 & 0,0 & $*$ \\
\hline $\mathrm{SB}^{1}$ & $2,7 \mathrm{a}$ & 2,6 & 1,9 & 4,0 & 0,4 & 16,6 & 0,7 & 0,7 & $*$ \\
\hline $\mathrm{SB}^{2}$ & $2,2 b$ & 2,0 & 0,8 & 4,0 & 0,7 & 33,7 & 0,5 & 0,1 & $*$ \\
\hline $\mathrm{CTC}^{1}$ & $8,0 \mathrm{~b}$ & 8,1 & 6,7 & 8,9 & 0,5 & 6,7 & $-0,3$ & $-0,4$ & $*$ \\
\hline $\mathrm{CTC}^{2}$ & $9,0 \mathrm{a}$ & 9,1 & 8,0 & 10,8 & 0,6 & 6,5 & 0,9 & 2,1 & $*$ \\
\hline $\mathrm{V}^{1}$ & $33,9 \mathrm{a}$ & 32,8 & 24,8 & 55,0 & 6,1 & 18,1 & 1,1 & 1,8 & $*$ \\
\hline $\mathrm{V}^{2}$ & $24,4 b$ & 22,2 & 8,5 & 47,7 & 8,9 & 36,4 & 0,5 & 0,1 & $*$ \\
\hline
\end{tabular}

${ }^{1}$ : profundidade de 0-0,05 m; ${ }^{2}$ : profundidade de 0,05-0,15 m; Min.: valor mínimo; Máx.: valor máximo; S: desvio-padrão; CV: coeficiente de variação; Cs: coeficiente de assimetria; Ck: coeficiente de curtose; ns: distribuição normal pelo teste Kolmogorov-Smirnov (p $\leq 0,05$ ) e *: distribuição não definida. Para um mesmo atributo, médias seguidas de mesma letra minúscula, em coluna, não diferem significativamente ( $\mathrm{p} \leq 0,05)$

do adubo (11-52-00) na linha de semeadura adicionado abaixo de $0,05 \mathrm{~m}$ de profundidade pelo mecanismo de adubação, além da baixa mobilidade do fósforo no solo. Segundo Silva et al. (2010a) a disponibilidade de fósforo no solo está relacionada com os teores de argila e de areia sendo essa reduzida em situações nas quais os teores de argila são elevados, como acontece no solo desse estudo.

$\mathrm{O}$ pH indica solo ácido nas duas camadas. Esses resultados estão associados, principalmente, à ausência de calagem e, em menor proporção, à lenta mineralização da MO, tanto em superfície quanto em profundidade, contribuindo, assim, para a liberação de ácidos orgânicos. De acordo com Tekest et al. (2009), em sistemas de plantio direto é comum a reduzida mineralização da matéria orgânica, associada à valores reduzidos de $\mathrm{pH}$.

Apesar dos valores de acidez elevados, observa-se baixo valor de $\mathrm{Al}$ em solução. Tal valor encontrado tem relação com o material de origem e a presença de MO, que possui a capacidade de complexar o $\mathrm{Al}$, reduzindo seu efeito tóxico às plantas (CELIK; BOYDAS; ALTIKAT, 2011).

$\mathrm{Na}$ ACP (Tabela 2), realizada para os onze atributos originais estudados na camada de $0-0,05 \mathrm{~m}$, foram extraídos três componentes que, de forma acumulada, explicam 80,29\% da variabilidade total dos dados. Os demais, contribuíram de forma irrisória e, portanto, não foram considerados na análise.

A primeira componente principal (CP1) explica $49,31 \%$ da variabilidade total dos dados, apresentando correlação elevada com o pH, $\mathrm{SB}, \mathrm{Al}, \mathrm{H}+\mathrm{Al}$ e $\mathrm{V}$ e moderada com K, Ca e Mg (Tabela 3), sendo, portanto, relacionado à fertilidade do solo. A ausência de correlação desta primeira componente com o $\mathrm{P}$ pode estar relacionada à sua baixa disponibilidade nessa camada, a qual se justifica pela ausência de correção dos níveis do mesmo, devido à forma de aplicação e à baixa mobilidade deste nutriente. 
Tabela 2 - Resumo das componentes principais na camada de 0- 0,05 m

\begin{tabular}{lccc}
\hline CP & Autovalor & Porcentagem da variância & Porcentagem acumulada \\
\hline 1 & 5,42 & 49,31 & 49,31 \\
2 & 1,77 & 16,09 & 65,40 \\
3 & 1,64 & 14,88 & 80,29 \\
\hline
\end{tabular}

CP: componente principal

Tabela 3 - Correlações entre os atributos originais na camada de 0-0,05 $\mathrm{m}$ e as componentes principais

\begin{tabular}{lccccccccccc}
\hline Atributos & $\mathrm{pH}$ & $\mathrm{MO}$ & $\mathrm{P}$ & $\mathrm{K}$ & $\mathrm{Ca}$ & $\mathrm{Mg}$ & $\mathrm{Al}$ & $\mathrm{H}+\mathrm{Al}$ & $\mathrm{SB}$ & $\mathrm{CTC}$ & $\mathrm{V}$ \\
\hline CP 1 & $0,94^{*}$ & 0,01 & $-0,29$ & 0,75 & 0,88 & 0,76 & $-0,85$ & $-0,91$ & 0,84 & $-0,45$ & 0,96 \\
CP 2 & $-0,06$ & 0,81 & $-0,23$ & 0,09 & $-0,01$ & 0,55 & $-0,02$ & 0,38 & 0,29 & 0,72 & $-0,02$ \\
CP 3 & 0,12 & 0,35 & $-0,57$ & 0,09 & $-0,72$ & 0,13 & $-0,38$ & $-0,08$ & $-0,44$ & $-0,47$ & $-0,25$ \\
\hline
\end{tabular}

*Negrito é significativo $(\mathrm{p} \leq 0,05)$

A segunda componente principal (CP2) explica $16,09 \%$ da variabilidade total. Nessa componente, a MO apresentou alta correlação, bem como a CTC, o que mostra a relação entre esses atributos, ou seja, a CTC tende a ser maior em locais onde os teores de MO são igualmente maiores. Nos solos tropicais e altamente intemperizados, como os solos do Cerrado, a MO é um componente importante para fornecer pontos de carga negativa em quantidade superior aos disponibilizados pelos principais constituintes dos minerais de argila destes solos (oxihidróxidos de ferro e alumínio e caulinita), porque nos valores de $\mathrm{pH}$ das camadas superficiais a $\mathrm{MO}$ possui carga líquida negativa, tendo uma CTC maior do que a CTA.

A Tabela 4 apresenta as três componentes principais da análise multivariada, realizada para os onze atributos na camada de 0,05-0,15 m, que explicam conjuntamente $90,88 \%$ da variância total e a porcentagem da variância explicada por cada um deles.

A componente principal (CP1) explica $68,44 \%$ da variabilidade total dos dados, apresentando alta correlação com o pH, Ca, Mg, SB, Al, H+Al e V (Tabela 5) e moderada com CTC e K, indicando também baixo teor de fósforo nessa camada, como observado na camada de 0-0,05 m. No entanto, esses valores de $\mathrm{P}$ não são provenientes de ausência de adubação, uma vez que essa foi realizada no ato da semeadura e em profundidade. Igualmente ao observado na camada de 0-0,05 $\mathrm{m}$, esse componente corresponde à fertilidade do solo, a qual é, apesar de baixa, mais equilibrada em relação à camada superficial.

A componente principal dois (CP2) explica 13,08\% e apresenta moderada correlação com a MO e CTC, como ocorreu na camada de 0-0,05 m. Os valores de acidez também são elevados nesta camada, no entanto a CTC é classificada como média. Neste caso, esta sofre menor influência da MO, conforme observado nos valores de correlação entre a componente e a MO e CTC. Tal fato pode ser justificado pela menor quantidade de MO nesta camada em relação à camada mais superficial e pelas quantidades dos nutrientes catiônicos mais elevados nessa quando comparados à camada mais superficial.

Para as duas camadas estudadas, apenas dois componentes principais forneceram resultados interpretáveis, de acordo com os critérios estabelecidos para seleção. Neste caso, esses componentes são suficientes para avaliar a variabilidade espacial dos atributos químicos desse solo. Silva et al. (2010b) encontraram resultados semelhantes para um Latossolo Vermelho Amarelo húmico. De acordo com esses autores, um ou dois componentes são, geralmente, suficientes para explicar toda a variação contida nos atributos de solo.

Tabela 4 - Resumo dos componentes principais na camada de 0,05-0,15 m

\begin{tabular}{cccc}
\hline CP & Autovalor & Porcentagem da variância & Porcentagem acumulada \\
\hline 1 & 7,53 & 68,44 & 68,44 \\
2 & 1,44 & 13,08 & 81,52 \\
3 & 1,03 & 9,36 & 90,88 \\
\hline
\end{tabular}


Tabela 5 - Correlações entre os atributos originais na camada de $0,05-0,15 \mathrm{~m}$ e as componentes principais

\begin{tabular}{llllllllllll}
\hline Atributos & $\mathrm{pH}$ & $\mathrm{MO}$ & \multicolumn{1}{c}{$\mathrm{P}$} & $\mathrm{K}$ & $\mathrm{Ca}$ & $\mathrm{Mg}$ & $\mathrm{Al}$ & $\mathrm{H}+\mathrm{Al}$ & $\mathrm{SB}$ & \multicolumn{1}{c}{$\mathrm{CTC}$} & \multicolumn{1}{c}{$\mathrm{V}$} \\
\hline CP 1 & $0,98^{*}$ & 0,47 & 0,15 & 0,76 & 0,95 & 0,95 & $-0,89$ & $-0,94$ & 0,98 & $-0,55$ & 0,99 \\
CP 2 & 0,04 & $-0,77$ & $-0,04$ & $-0,25$ & $-0,01$ & $-0,17$ & $-0,25$ & $-0,31$ & $-0,14$ & $-0,75$ & $-0,01$ \\
CP 3 & 0,02 & $-0,15$ & 0,97 & $-0,11$ & 0,1 & $-0,07$ & $-0,06$ & 0,05 & 0,03 & 0,14 & 0,01 \\
\hline
\end{tabular}

*Negrito é significativo $(\mathrm{p} \leq 0,05)$

Tabela 6 - Modelos e parâmetros dos variogramas escalonados para as duas componentes principais nas duas camadas

\begin{tabular}{lccccccc}
\hline \multicolumn{1}{c}{ Atributos } & Modelo & $\mathrm{C}_{0}$ & $\mathrm{C}_{0}+\mathrm{C}$ & $\mathrm{a}(\mathrm{m})$ & $\mathrm{IDE}$ & $\mathrm{R}^{2}$ & $\mathrm{~S} . \mathrm{VC}$ \\
\hline $\mathrm{CP} 1(0-0,05)$ & $\mathrm{ESF}$ & 0,36 & 1,13 & 10,0 & 32 & 90 & $*$ \\
$\mathrm{CP} 2(0-0,05)$ & $\mathrm{ESF}$ & 0,33 & 1,11 & 20,0 & 30 & 76 & $*$ \\
$\mathrm{CP} 1(0,05-0,15)$ & $\mathrm{ESF}$ & 0,51 & 1,08 & 17,0 & 47 & 50 & $*$ \\
$\mathrm{CP} 2(0,05-0,15)$ & $\mathrm{EXP}$ & 0,49 & 1,21 & 40,0 & 40 & 60 & $*$ \\
\hline
\end{tabular}

$\mathrm{C}_{0}$ : efeito pepita; $\mathrm{C}_{0}+\mathrm{C}$ : patamar; a: alcance; IDE: índice de dependência espacial; $\mathrm{R}^{2}$ : coeficiente de determinação e S.VC: correlação significativa entre os valores observados e os estimados pela validação cruzada $(\mathrm{p} \leq 0,05)$

Após a seleção dos componentes, os mesmos foram submetidos à análise geoestatística. Os resultados dos parâmetros e modelos da análise de dependência espacial nas duas camadas estudadas encontram-se na Tabela 6 .

O modelo que melhor se ajustou a componente um (CP1) e a dois (CP2) na camada de 0-0,05 m foi o esférico, com alcance de dependência espacial de 10 e $20 \mathrm{~m}$, respectivamente. Na camada de 0,05-0,15 m, a CP1 ajustouse ao modelo esférico com alcance de $17 \mathrm{~m}$ e a CP2 ajustou-se ao modelo exponencial com alcance de $40 \mathrm{~m}$ (Figura 1).

$\mathrm{O}$ índice de dependência espacial para as componentes nas duas camadas foi moderado, com variação entre $25 \%$ a $75 \%$, conforme classificação proposta por Cambardella et al. (1994).

As CP2 nas duas camadas estão relacionadas fortemente com a MO e com a CTC apresentando, assim, maior continuidade espacial observada pelos maiores valores de alcance para essas componentes. Isso se deve ao não revolvimento do solo para a semeadura e à lenta decomposição dos resíduos orgânicos, indicando que a CTC e a MO apresentam maior continuidade espacial na área em relação aos indicadores da fertilidade do solo, ou seja, pontos amostrais que se encontram a uma distância menor que os alcances determinados são correlacionados entre si e maior é a precisão na estimação de valores em locais não amostrados.

Maior continuidade espacial foi também observada para a camada de 0,05-0,15 m quando comparada à camada de 0-0,05 m. Apesar dessa maior continuidade, representada por áreas mais homogêneas, conforme observado nos mapas das Figuras 2 e 3, a fertilidade do solo de modo geral foi inferior.

Nas Figuras 2 e 3 estão os mapas construídos por krigagem ordinária com base nos parâmetros e modelos dos variogramas ajustados para as CP1 e CP2 nas camadas de 0-0,05 m e 0,05-0,15 m. Verifica-se, conforme discutido, maior continuidade espacial na camada de 0,05-0,15 m,

Figura 1 - Variogramas escalonados para a componente principal um (CP1) e a dois (CP2) nas camadas de 0-0,05 m e 0,05-0,15 m
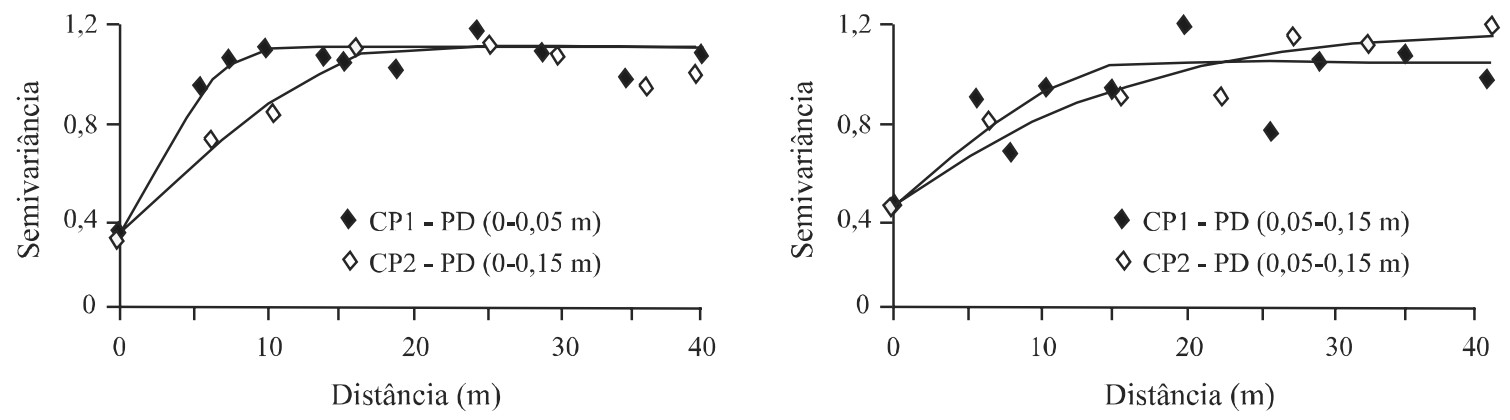
Figura 2 - Mapas da primeira (A) e segunda (B) componente principal (CP1 e CP2) construídos com base nos atributos químicos do solo na profundidade de $0-0,05 \mathrm{~m}$

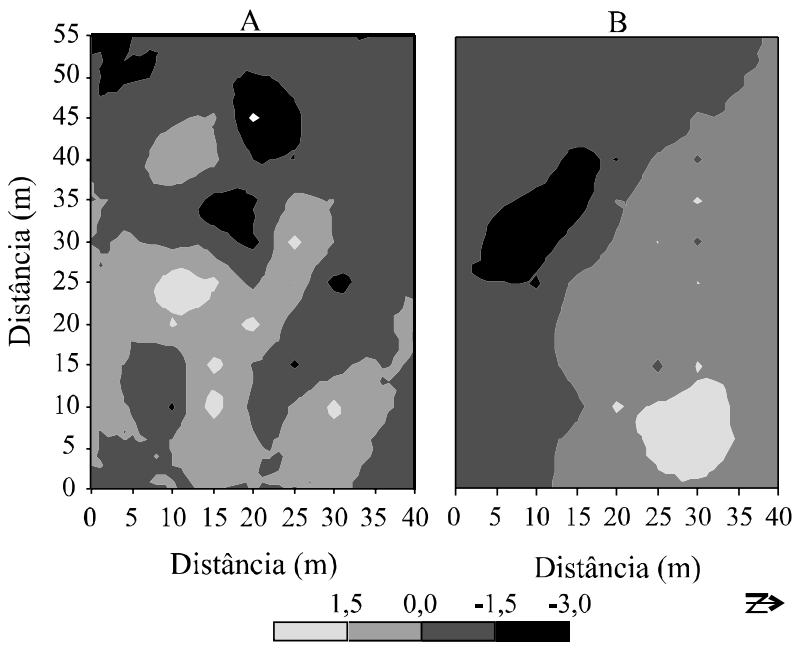

com a formação de "zonas de fertilidade" (áreas de mesma cor) mais bem definidas.

Os valores de fertilidade química do solo na camada de 0-0,05 m são maiores na maior parte da área (Figura 2A), com as maiores concentrações na região lateral esquerda da mesma (valores positivos dos escores).

Analisando a Figura 2B, observa-se que os níveis de CTC e a MO possuem menor variabilidade horizontal com os maiores valores na região superior e lateral esquerda da área e menores valores na região inferior. Essa íntima relação entre CTC e MO devese ao fato de que a MO sofre desprotonação de seus grupamentos carboxílicos e fenólicos, aumentando a carga negativa e, deste modo, eleva a CTC mesmo em solos de baixa fertilidade. $\mathrm{O}$ aumento nos estoques de $\mathrm{MO}$ em plantio direto é suficiente para ocasionar aumento expressivo na CTC destes solos (CELIK; BOYDAS; ALTIKAL, 2011; CIOTTA et al., 2002; TOPAKCI et al. 2011). Esse aumento é observado, principalmente, na camada de 0-0,05 m, uma vez que na camada de 0,05-0,15 m (Figura 3B), os teores de MO foram menores e a sua relação com a CTC também foi menos expressiva.

A adubação na semeadura proporcionou melhoria nos níveis dos nutrientes na camada de 0,05$0,15 \mathrm{~m}$ (Figura 3B). Maior parte da área encontrase com valores positivos indicando fertilidade mais equilibrada do solo na sua distribuição espacial nesta camada quando comparado com a camada mais superficial.
Figura 3 - Mapas referentes à primeira (A) e à segunda (B) componente principal gerados com base nos atributos químicos do solo na profundidade de $0,05-0,15 \mathrm{~m}$

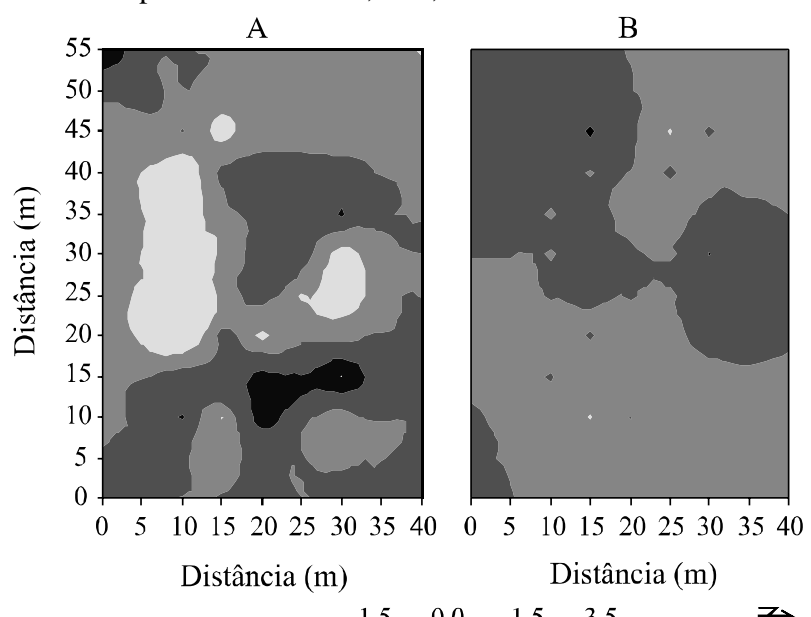

\section{CONCLUSÕES}

1. A análise multivariada, associada à análise geoestatística, apresentou resultados satisfatórios da distribuição espacial da fertilidade;

2. A matéria orgânica apresentou maior continuidade espacial dada às características do sistema de plantio direto;

3. De maneira geral, a área apresentou baixa fertilidade para o cultivo da soja, com diferente padrão de distribuição espacial entre camadas.

\section{REFERÊNCIAS}

AMADO, T. J. C. et al. Variabilidade espacial e temporal da produtividade de culturas sob sistema plantio direto. Pesquisa Agropecuária Brasileira, v. 42, n. 08, p. 11011110, 2007.

AZEVEDO, D. M. P. et al. Atributos físicos e químicos de um Latossolo Amarelo e distribuição do sistema radicular da soja sob diferentes sistemas de preparo no cerrado maranhense. Revista Ciência Agronômica, v. 38, n. 01, p. 32-40, 2007.

CAMBARDELLA, C. A. et al. Field-scale variability of soil properties in central Iowa soils. Soil Science Society American Journal, v. 58, n. 05, p. 1501-1511, 1994.

CARVAlHO, J. R. P.; SILVEIRA, P. M.; VIEIRA, S. R. Geoestatística na determinação da variabilidade espacial de características químicas do solo sob diferentes preparos. Pesquisa Agropecuária Brasileira, v. 37, n. 08, p. 1151-1159, 2002.

CELIK, A.; BOYDAS, M. G.; ALTIKAT, S. A comparison of an experimental plow with a moldboard and a disk plow on the soil 
physical properties. Applied Engineering in Agriculture, v. 27, n. 02, p. 185-192, 2011.

CIOTTA, M. N. et al. Acidificação de um Latossolo sob plantio direto. Revista Brasileira de Ciência do Solo, v. 26, p. 1055-1064, 2002.

EMPRESA BRASILEIRA DE PESQUISA AGROPECUÁRIA. Centro Nacional de Pesquisa de Solos. Manual de métodos de análise de solo. 2. ed. Rio de Janeiro, 1997. 212 p.

EMPRESA BRASILEIRA DE PESQUISA AGROPECUÁRIA. Centro Nacional de Pesquisa de Solos. Sistema Brasileiro de Classificação de Solos. 2. ed. Rio de Janeiro, 2006. 412 p.

GREGO, C. R. et al. Geostatistical analysis for soil moisture content under the no tillage cropping system. Scientia Agricola, v. 63, n. 04, p. 341-350, 2006.

GREGO, C. R.; VIEIRA, S. R. Variabilidade espacial de propriedades físicas do solo em uma parcela experimental. Revista Brasileira de Ciência do Solo, v. 29, p. 169-177, 2005.

ISAAKS, E. H.; SRIVASTAVA, M. An introduction to applied geoestatistics. New York: Oxford University Press, 1989. 560 p.

JOHNSON, R. A.; WICHERN, D. W. Applied multivariate statistical analysis. 5. ed. New Jersey: Prentice Hall, 2002. 767 p.

KENT, M.; COKER, P. Vegetation description and analysis. Baffins Lane: John Wiley \& Sons, 1992. 363 p.

LEÃO, A. B. et al. Variabilidade espacial de fósforo em solo do perímetro irrigado Engenheiro Arcoverde, PB. Revista Ciência Agronômica, v. 38, n. 01, p. 01-06, 2007.

LIBARDI, P. L. et al. Variabilidade da umidade gravimétrica de um solo hidromórfico. Revista Brasileira de Ciência do Solo, v. 20, n. 01, p. 1-12, 1996.

MOTTA, P. E. F. et al. Ocorrência de macaúba em Minas Gerais: relação com atributos climáticos, pedológicos e vegetacionais. Pesquisa Agropecuária Brasileira, v. 37, n. 07, p. 1023-1031, 2002.
RAIJ, B. et al. Análise química para avaliação da fertilidade de solos tropicais. Campinas: Instituto Agronômico, 2001. 285 p.

SECCO, D. et al. Produtividade de soja e propriedade física de um Latossolo submetido a sistema de manejo e compactação. Revista Brasileira de Ciência do Solo, v. 28, n. 04, p. 797-804, 2004.

SILVA, V. R.; REICHERT, J. M.; REINERT, D. J.; Variabilidade espacial da resistência do solo à penetração em plantio direto do solo à penetração em plantio direto. Ciência Rural, v. 34, n. 02, p. 399-406, 2004.

SILVA, S. A. et al. Variabilidade espacial de atributos químicos de um Latossolo Vermelho-Amarelo húmico cultivado com café. Revista Brasileira de Ciência do Solo, v. 34, p. 15-22, 2010 b.

SILVA, S. A. et al. Variabilidade espacial do fósforo e das frações granulométricas de um Latossolo Vermelho Amarelo. Revista Ciência Agronômica, v. 41, n. 01, p. 01-08, 2010a.

STRECK, C. A. et al. Modificações em propriedades físicas com a compactação do solo causada pelo tráfego induzido de um trator em plantio direto. Ciência Rural, v. 34, n. 03, p. 755-760, 2004.

TEKEST, M. Z. et al. Soil drying effects on spatial variability of soil hardpan attributes on pacolet sandy loam soil. Transactions of the ASABE. v. 52, n. 03, p. 697-705, 2009.

TOPAKCI, M. et al. Sesame hill dropping performance of a vacuum seeder for different tillage practices. Applied Engineering in Agriculture, v. 27, n. 02, p. 203-209, 2011.

WANG, Y.; CHEN, Y.; RAHMAN, S. Tillage effects on soil strength and crop growth for Red River clay. The Canadian Society for Bioengineering, v. 202, n. 06, p. 1-13, 2006.

WARRICK, A. W.; NIELSEN, D. R. Spatial variability of soil physical properties in the field. In: HILLEL, D. (Ed.). Application of soil physics. New York: Academic Press, 1980. p. 319-344.

ZWICK, W. R.; VELICER, W. F. Factors influencing four rules for determining the number of components to retain. Psychological Bulletin, v. 99, n. 03, p. 432 - 442, 1986. 